\title{
Abnormal Cardiac Strain in Children and Young Adults with HIV Acquired in Early Life
}

\author{
Amy Sims, MD, Lowell Frank, MD, Russell Cross, MD, Sarah Clauss, MD, David Dimock, \\ BA, Julia Purdy, CPNP, Irene Mikhail, MD, Rohan Hazra, MD, Colleen Hadigan, MD, MPH, \\ and Craig Sable, MD \\ Department of Cardiology, Children's National Medical Center, Washington, District of Columbia \\ (A.S., L.F., R.C., S.C., C.S.); the Laboratory of Immunoregulation, National Institute of Allergy and \\ Infectious Diseases, Bethesda, Maryland (D.D., I.M., C.H.); the Critical Care Medicine \\ Department, Clinical Center (J.P.), HIV and AIDS Malignancy Branch (J.P., R.H.), National \\ Cancer Institute, Bethesda, Maryland; and the Pediatric, Adolescent, and Maternal AIDS Branch, \\ Eunice Kennedy Shriver National Institute of Child Health and Human Development, National \\ Institutes of Health, Bethesda, Maryland (R.H.)
}

\begin{abstract}
Background-Traditional measures of cardiac function are now often normal in adolescents and young adults treated with antiretroviral therapy for human immunodeficiency virus (HIV) infection. There is, however, evidence of myocardial abnormalities in adults with HIV. Cardiac strain analysis may detect impairment in cardiac function that may be missed by conventional measurements in this population.
\end{abstract}

Methods-This was a retrospective study in which echocardiograms of HIV-infected subjects ( $n$ $=28$ ) aged 7 to 29 years who participate in a natural history study of HIV acquired early in life were analyzed and compared with matched controls. Standard echocardiographic measures, along with speckle tracking-derived strain and strain rate, were assessed.

Results-Among the HIV-infected subjects, the median CD4 count was 667 cells $/ \mathrm{mm}^{3}$, and the mean duration of antiretroviral therapy was 14.6 years. Ejection fractions and fractional shortening were normal. There were no significant differences in measures of systolic or diastolic function between the groups. The HIV-infected group had borderline increased left ventricular mass indices. Global longitudinal and circumferential strain and strain rate, as well as global radial strain rate, were significantly impaired in the HIV-infected group compared with controls. There were no associations identified between left ventricular mass index or strain indices and current CD4 count, CD4 nadir, HIV viral load, or duration of antiretroviral therapy.

Conclusions-HIV-infected participants demonstrated impaired strain and strain rate despite having normal systolic function and ejection fractions. Strain and strain rate may prove to be prognostic factors for long-term myocardial dysfunction. Therefore, asymptomatic children and young adults with long-standing HIV infection may benefit from these more sensitive measures.

\section{Keywords}

Echocardiography; Strain; HIV; Children

\footnotetext{
Copyright 2012 by the American Society of Echocardiography.

Reprint requests: Colleen Hadigan, MD, MPH, National Institute of Allergy and Infectious, Diseases, Laboratory of Immunoregulation, 10 Center Drive, Building 10, Room 11C103, Bethesda, MD 20892 (hadiganc@ niaid.nih.gov).
} 
It is known that people living with human immunodeficiency virus (HIV) have a high risk for cardiac disease, including pericarditis, myocarditis, cardiomyopathy, pulmonary hypertension, and valvular disease. ${ }^{1,2}$ Cardiac dysfunction has also been extensively described in children with HIV, but much of the cardiac dysfunction was described before the availability of highly active antiretroviral therapy (ART). Even asymptomatic children not on highly active ART have been shown to have cardiac abnormalities on echocardiography. In a study of 26 Indian children aged 1 to 9 years with perinatally acquired HIV not yet on highly active ART, Shah et al. ${ }^{3}$ found that $77 \%$ had evidence of cardiac abnormalities on echocardiography. The most common abnormalities found were left ventricular (LV) dilation and LV hypertrophy. ${ }^{3}$ Cunha et al. ${ }^{4}$ found that LV dysfunction was common in Brazilian children approximately 18 months after acquired immune deficiency syndrome diagnosis. Their data suggest a much lower incidence of LV dysfunction in patients treated with ART. They also suggest that the observed decreased in cardiac disease with ART may in fact be a delay in the onset of cardiovascular manifestations of HIV. ${ }^{4}$

With the advent of potent ART, there has been a shift from significant cardiac disease thought to be a result of opportunistic infections or the HIV virus to seemingly few cardiac complications in the pediatric population. Adult studies have shown that asymptomatic HIVinfected patients do manifest mild functional and morphologic cardiac abnormalities. ${ }^{5,6}$ In addition, lipid and metabolic derangements have also been commonly found in the HIVinfected population, both with and without ART. ${ }^{7}$

Echocardiographic measures of strain and strain rate are more sensitive indicators of function than the traditional measures of ejection fraction and shortening fraction and may provide a means for the early detection of cardiac dysfunction. ${ }^{8}$ Echocardiographic strain analysis uses speckle tracking to track the displacement and velocities of acoustic markers of individual segments of the myocardium as they move in different vectors. Speckles are areas of scattering, reflection, and interference in the myocardium caused by the ultrasound beam that can be tracked frame by frame through the cardiac cycle. ${ }^{9-11}$ Strain measures the regional fractional shortening of the myocardium, and strain rate is the integration of strain over time. Speckle tracking-derived strain rate is particularly useful because it is less likely to be preload and afterload dependent and is angle independent. Multiple studies have demonstrated the accuracy and reliability of strain and strain rate in the assessment of myocardial function. ${ }^{12-18}$ Global strain has been validated in the pediatric population using tagged magnetic resonance imaging as the gold standard. ${ }^{19}$

In addition, strain and strain rate imaging can detect subclinical myocardial dysfunction at an earlier stage compared with conventional imaging. ${ }^{20}$ Intraobserver and interobserver variability in strain parameters has been measured and validated in previous studies. ${ }^{21}$

We hypothesized that strain analysis may detect preclinical or asymptomatic cardiac abnormalities in pediatric HIV-infected populations. The aim of our study was to determine if there are differences in strain and strain rate between patients who acquired HIV in early life and matched controls. A secondary aim was to evaluate potential relationships between cardiac strain parameters and markers of immunologic status, inflammation, and cardiac dysfunction.

\section{METHODS}

Echocardiograms were obtained from 28 children and young adults aged 7 to 29 years with known HIV disease and followed in a natural history protocol of HIV acquired in infancy or early childhood at the National Institutes of Health (NIH). Echocardiography was performed 
as part of an established research protocol, not because of symptoms or comorbidities. At the time this study was completed, 32 participants had echocardiograms available for analysis, but two studies were excluded for technical limitations and two for preexisting congenital heart defects. Because of the retrospective nature of the study, the use of M-mode echocardiography was not consistently collected for all patients.

The echocardiograms were obtained between September 2008 and August 2009, but were analyzed in a retrospective manner for this nested study. All NIH subjects and/or their guardians, as appropriate, completed written informed consent.

Control data were obtained by querying the echocardiographic database at the Children's National Medical Center for "no cardiac disease identified." From the list generated from the this query, control subjects $(n=28)$ were age and gender matched to the HIV-infected participants. Control subjects were excluded if they had known exposure to chemotherapeutic medications, underwent more than one echocardiographic study, or had known genetic syndromes as the indications for study. The institutional review boards of Children's National Medical Center and the National Institute of Allergy and Infectious Diseases approved this study.

\section{Echocardiographic Measurements}

Echocardiographic measurements were performed retrospectively on subject and control echocardiograms. Traditional measures of LV systolic function such as ejection fraction and fractional shortening, as well as indicators of diastolic function such as mitral inflow E/A ratio and tissue Doppler-derived E/ $\mathrm{E}^{\prime}$ ratio, were evaluated. $\mathrm{LV}$ ejection fraction was calculated using the single-plane modified Simpson's rule in the four-chamber view. LV mass was calculated from M-mode tracings using the method of the American Society of Echocardiography in the short-axis view. Tricuspid regurgitation velocity as an indicator of pulmonary artery pressure was also measured. Tissue Doppler velocities were measured from the septal annulus from a single representative beat. All measurements were performed by a pediatric cardiologist according to American Society of Echocardiography standards. ${ }^{22}$

Strain measurements were performed using Syngo Velocity Vector Imaging software (Siemens Healthcare, Erlangen, Germany) on Digital Imaging and Communications in Medicine standard digital echocardiograms for each HIV and control subject. Measurements of strain by speckle-tracking imaging were recorded in all 56 patients (28 HIV-infected patients and 28 controls). Each study was analyzed from the parasternal short-axis and apical four-chamber views (Figures 1 and 2). Each tracing was performed during endsystole, and the short-axis view was analyzed at the level of the papillary muscles. Each tracing was repeated twice, and measurements from the two best tracking images were averaged for each strain parameter. Six segments were used to calculate global strain from the short-axis and apical four-chamber views.

Traditional echocardiographic and strain measurements were performed by a single cardiologist on both the patients with HIV and the controls. The reader was blinded to the clinical characteristics of the study population but was not completely blinded to the study groups, because of technical differences in the acquisition of some of the studies. Although all of the studies were deidentified, some of the study echocardiograms obtained at the NIH did not apically invert the four-chamber view, whereas all of the four-chamber views of the control echocardiograms were apically inverted. Ten randomly selected echocardiograms (five from each group) were reanalyzed for interobserver variability. The second reader, who was blinded to study group, made independent measurements, which were then correlated with the initial reader's analysis. Intraobserver variability was determined using the correlation coefficient between the first reader's two analyses of each echocardiogram. 
Short-axis measurements included circumferential strain (\%), radial strain (\%), circumferential strain rate $\left(\mathrm{sec}^{-1}\right)$, and radial strain rate $\left(\mathrm{sec}^{-1}\right)$. Longitudinal strain $(\%)$ and longitudinal strain rate $\left(\mathrm{sec}^{-1}\right)$ were recorded from the apical four-chamber view (Figures 1 and 2).

Strain is the percentage of deformation of the myocardium. This percentage is measured by continuously tracking natural acoustic markers (speckles) frame by frame and calculating the end-systolic distance between two particles (or speckles) of tracked myocardium minus the original distance between those two particles all over the original length. In the longitudinal and circumferential directions, speckles of endocardium are tracked. The myocardium contracts in these directions during systole, so these values will be negative percentages. Radial strain measures the percentage of deformation in the radial direction, so both the endocardium and epicardium are tracked to calculate radial strain. The myocardium thickens in the radial direction during systole, so radial strain is measured as a positive percentage.

\section{Laboratory Assays}

Laboratory measurements were performed on the HIV-infected study patients as part of the NIH study protocol and analyzed at the central NIH laboratory in Bethesda, Maryland, and included CD4 T-cell count, HIV viral ribonucleic acid by reverse transcription polymerase

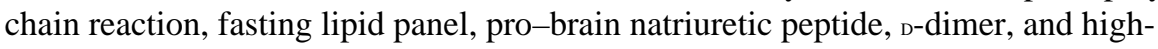
sensitivity C-reactive protein.

\section{Statistical Analysis}

Direct group comparisons were performed using Wilcoxon's rank-sum analyses. Correlations within the HIV-infected study group were found using Pearson's coefficient of correlation analysis. A multivariate regression analysis was performed to evaluate the association between strain measures and age, race, sex, body mass index, blood pressure, lipids, and duration of ART. Intraobserver and interobserver variability was evaluated through intraclass correlation coefficients calculated from available measurements. Statistical significance was assumed for $P$ values $<.05$.

\section{RESULTS}

HIV-infected subjects ranged in age from 7 to 29 years. All HIV-infected study participants acquired HIV from mother-to-child transmission, except for two participants who acquired HIV from blood transfusions performed in infancy. Control participants ranged from 7 to 32 years of age. There were no significant differences between the percentage of Caucasian and non-Caucasian subjects and the percentage of African American subjects between the HIV and control groups. There were more Hispanic subjects in the control group than in the HIVinfected group. In addition, there was a trend toward statistical significance in blood pressure between groups (Table 1), with the HIV-infected group having higher blood pressures. All HIV-infected participants were asymptomatic from a cardiovascular standpoint. The primary indications for control echocardiographic studies were chest pain ( $n$ $=13)$ or syncope $(n=10)$ in the outpatient clinic setting. Other indications included abnormal chest x-ray $(n=2)$, murmur $(n=2)$, abnormal electrocardiographic findings $(n=$ $1)$, palpitations $(n=1)$, and headaches $(n=1)$. Some participants had two indications for echocardiography.

\section{Echocardiographic Findings}

Echocardiographic parameters are summarized in Table 1. Echocardiographic measurements of chamber size, systolic and diastolic function, and estimated pulmonary artery systolic 
pressure were within normal limits in control and HIV subjects. Ejection fraction and measures of diastolic function were not significantly different between the HIV-infected and control groups. Fifteen of the $28 \mathrm{HIV}$-infected participants underwent M-mode echocardiography as part of their routine studies. There was a trend toward higher shortening fractions $(P=.06)$ and higher LV mass indices (LVMIs) $(P=.06)$ in HIVinfected subjects. In addition, mean LV mass $Z$ scores were significantly different between groups, with the HIV-infected subjects having greater LV mass $Z$ scores $(-0.51 \pm 1.11$ vs $1.04 \pm 0.61, P=.046$ ) than control subjects (Table 1).

The HIV-infected group had significantly impaired strain indices compared with controls (Figure 3). Longitudinal strain $(P<.001)$, longitudinal strain rate $(P<.001)$, circumferential strain $(P<.001)$, circumferential strain rate $(P<.001)$, and global radial strain rate $(P=$. $001)$ were significantly decreased compared with controls. Radial strain was not found to be different between the groups. The strain measurements in both the study and control population were less than reported normal ranges. ${ }^{13}$ For longitudinal strain, $96 \%$ of the HIV-infected participants had abnormal values (below the fifth percentile), and $55 \%$ of the control participants were below the fifth percentile. For radial strain, $92 \%$ of the HIVinfected and $80 \%$ of the control participants had values below the fifth percentile. For circumferential strain, $37 \%$ of the HIV-infected and $4 \%$ of the control participants had values below the fifth percentile, whereas $4 \%$ of the HIV-infected and $15 \%$ of controls were greater than 95 th percentile. ${ }^{13}$

Three of the HIV-infected participants had histories of B-cell lymphoma and had received chemotherapy. Group comparisons excluding these three subjects did not change any of the significant differences between groups with regard to strain parameters. Frame rates of both HIV-infected and control subjects ranged between 25 and 60 frames/sec for all studies.

With the exception of intraobserver variability for radial strain and radial strain rate, strain measurements had very good agreement, with interobserver and intraobserver measurements based on intraclass correlation coefficients (Table 2).

\section{HIV-Associated Characteristics}

The median CD4 count was 667 cells $/ \mathrm{mm}^{3}$, and the mean duration of ART was 14.6 years. No association was found between measures of systolic or diastolic function or strain measures and current CD4 count, CD4 nadir, HIV viral load, nucleoside reverse transcriptase inhibitor exposure, protease inhibitor exposure, or duration of ART. There was also no correlation found between echocardiographic measures and selected biomarkers and inflammatory markers, such as brain natriuretic peptide, lipid profile, and high-sensitivity Creactive protein. In addition, there was no correlation with smoking status of the HIVinfected group. In multivariate regression analyses that included age, sex, race, body mass index, blood pressure, high-density lipoprotein and low-density lipoprotein cholesterol, and years of ART, age was identified as a significant predictor of longitudinal strain $(P=.04)$, but no other associations were identified with measures of strain.

Of the HIV-positive study population, $11 \%$ (three of 28) had high total cholesterol (>200 $\mathrm{mg} / \mathrm{dL}$ ), $29 \%$ (eight of 28 ) had high triglyceride levels ( $>150 \mathrm{mg} / \mathrm{dL}$ ), 29\% had low highdensity lipoprotein levels $(<40 \mathrm{mg} / \mathrm{dL}$ ), and $11 \%$ (three of 28 ) had high low-density lipoprotein levels ( $>130 \mathrm{mg} / \mathrm{dL}$ ). Two subjects had marked fasting hypertriglyceridemia (one subject had a value $>500 \mathrm{mg} / \mathrm{dL}$, and one subject had a value $>800 \mathrm{mg} / \mathrm{dL}$ ). No laboratory data were available for the control population.

There was an association between higher pulmonary pressure estimates and duration of exposure to zidovudine $(r=0.55, P=.004)$. All study patients had normal pulmonary 
pressure estimates. We also found that duration of exposure to tenofovir was associated with favorable measures of circumferential strain $(r=-0.40, P=.04)$, circumferential strain rate $(r=-0.42, P=.03)$, and radial strain rate $(r=0.70, P<.001)$. Thirteen of the $28 \mathrm{HIV}$ infected participants had never been exposed to tenofovir.

\section{DISCUSSION}

We studied a unique population of children and young adults with HIV acquired at a young age. This population has not previously been extensively studied in terms of echocardiographic findings in the era of effective HIV therapy. Deterioration of standard echocardiographic measures of ejection fraction and fractional shortening is often a late measure of cardiac dysfunction. Strain and strain rate are sensitive new measures of function that can give us insight into whether these patients are at higher risk for cardiovascular disease than their uninfected peers, despite being adequately treated with antiretroviral medications and clinically asymptomatic.

Compared with their uninfected peers in our study, however, HIV-infected participants demonstrated impaired (less negative) longitudinal and circumferential strain and strain rate and impaired (less positive) radial strain rate despite normal measures of systolic and diastolic function. No significant difference was found between HIV-infected participants and controls in radial strain. Radial strain measurement has more sources of error and is less validated than strain measurements in the other directions, because it incorporates measurements from the endocardium and epicardium. Because two surfaces are traced, and the true epicardium may be cut off in some studies, radial strain is not as reproducible a measure as strain measured in other vectors. ${ }^{23}$

Because it is a relatively new technology, there are limited established normal range values for strain and strain rate in this age group. The strain measurements in both the study and control populations were less than reported normal ranges. ${ }^{13}$ This may be due to differences between the various systems used to generate strain data from one study to the next. ${ }^{13,23}$ Frame rate differences may have the potential to alter strain results at very low or high frame rates, but all studies were performed within a range such that this was unlikely to happen $\left(25-60\right.$ frames/sec) ${ }^{24}$

Strain and strain rate are relatively new measures of cardiac function, and the exact clinical implications of decreased strain and strain rate are unknown. Previous studies have demonstrated that in certain populations, impaired strain and strain rate do carry a higher rate of mortality. ${ }^{25}$ Therefore, strain and strain rate may be prognostic factors for myocardial dysfunction. Impaired strain and strain rate may indicate early cardiovascular dysfunction and may represent premature aging in this population. Strain rate is a particularly useful and sensitive measure of function because it is likely less preload and afterload dependent and is angle independent.

Further study is warranted to determine the etiology of the difference in myocardial strain between the HIV-infected study population and control subjects. It is difficult to determine the exact etiology of possible subclinical myocardial dysfunction in our study population. The cause of subtle myocardial damage may be multifactorial. Causes may include metabolic disturbances observed in this population, such as hyperlipidemia and insulin resistance, antiretroviral medication toxicity including in utero exposure, chemotherapy, or myocardial invasion of the HIV virus itself. Many of the HIV-infected study subjects had abnormal lipid profiles, which have the potential contribute to the difference in strain parameters. Elevated lipids levels are a well-known characteristic of HIV-infected populations. Before the introduction of antiretroviral agents, elevated triglyceride and low- 
density lipoprotein cholesterol levels were reported with HIV in adults. After the initiation of ART including protease inhibitors, lipid levels may increase by $20 \%$ to $50 \%$ in HIVinfected children. ${ }^{7}$ Because the control population for this study was a retrospective procurement of normal echocardiograms in healthy children, lipid levels and other risk factors were not available for comparison.

In addition to difference in strain, LV mass as measured by LVMI and LV mass $Z$ score tended to be increased in patients with HIV compared with control subjects. Increased LVMI has been demonstrated in previous studies of cardiac features in asymptomatic adults and children with HIV. ${ }^{5}$ Prior studies have shown higher rates of diastolic dysfunction among adults with HIV, 5,6 which was not replicated in our study. This may be related to the relative young age of our study group or to our limited sample size. Decreased function has been noted in children exposed to ART in utero. ${ }^{26}$ It is unlikely that our population was exposed to ART prenatally, given that most were born before the widespread use of ART in pregnancy or were infected by transfusions during the first year of life.

It is important to consider why our HIV-infected study group had slightly higher LVMIs and $\mathrm{LV}$ mass $Z$ scores than controls and whether this could explain the impaired strain indices we observed. Even though not statistically significant, HIV-infected participants tended to have higher blood pressures. It is possible that early coronary artery disease, which is increased or accelerated in HIV, ${ }^{27}$ may contribute to both increased LVMI and impaired strain indices in this population. Alternatively, children and young adults with lifelong HIV infection may have experienced myocardial insults earlier in life that resulted in subtle scarring and subsequent impaired strain indices. It is important to also note that increased LVMI has been associated with increased mortality in HIV-infected children. ${ }^{28}$

One may expect that with impaired global strain in both the longitudinal and circumferential directions, a decrease in ejection fraction would be observed. This was likely not the case, however, because strain measurements derived from speckle tracking of the myocardium are able to detect much more subtle changes in myocardial function than ejection fraction and shortening fraction. Both ejection fraction and shortening fraction rely on geometric assumptions and are more prone to human error than speckle tracking, thus making strain measurements derived from speckle tracking a more accurate measure of function.

In this relatively small group of subjects, impaired strain and strain rate did not appear to be directly correlated with immunologic measures such as CD4 count or viral load, nor did we see any correlation between certain biomarkers such as lipids, C-reactive protein, and brain natriuretic peptide. There was a correlation between higher pulmonary artery pressure as estimated by tricuspid regurgitation velocity and time on zidovudine. A recent study demonstrated a link between zidovudine and other antiretroviral medications and pulmonary artery endothelial abnormalities in animal and in vitro assays, suggesting that zidovudine and other ART agents may contribute to the high incidence of pulmonary artery hypertension in HIV-infected patients. ${ }^{29}$ The estimated pulmonary pressures of all of the HIV-infected subjects were normal, so the significance of this observation is unclear. We also found that increased exposure to tenofovir was associated with more favorable strain indices. Associations such as these cannot be used to presume causality and must be interpreted with caution given the small sample size. Indeed, in a large adult cohort study, recent tenofovir exposure was related to an increased risk for heart failure, which is contradictory to our findings. ${ }^{30}$ The question of whether tenofovir provides a cardiotoxic or cardioprotective effect warrants further in-depth study. 


\section{Study Limitations}

Limitations of the current study included the limited sample size and the relatively wide age range of the HIV-infected participants. The study was completed by performing retrospective strain analysis on a convenience sample. Therefore, the echocardiograms were not acquired for this particular study, and some echocardiograms were missing M-mode views. In addition, the reader was not completely blinded, as some of the echocardiograms of HIV-positive patients obtained at the NIH did not apically invert the study, whereas all of the four-chamber views performed at the Children's National Medical Center were apically inverted. Another limitation is the difference in Hispanic ethnicity between the groups, although both groups were similar with regard to African American race. Prospective studies with larger sample sizes are needed to confirm our conclusions.

In addition, limited data were available for the control group, and it is possible that controls may not represent a completely healthy population. Another potential limitation is that echocardiograms for the HIV-infected participants and the controls were obtained at different centers. However, all echocardiograms were performed by trained echocardiographic technicians, and all strain analysis was centralized. Strain analysis software has a sophisticated algorithm, which limits observer bias, and we were able to demonstrate high intraobserver and inter observer correlations. Radial strain was not different between our two populations, but radial strain is the least validated strain measure and requires the tracing of both the endocardium and the epicardium, so more sources of error are introduced. Prior studies have shown minimal intraobserver and interobserver variability in the measurement of strain. ${ }^{31}$

Finally, strain technology is novel, and the clinical implications of these results are not yet completely known. We have observed a difference in strain, which has been shown in several populations to detect patients at risk for subsequent cardiac morbidity and mortality. Further study is needed to determine the significance of our findings in HIV-infected patients, but strain abnormalities may be an important preclinical precursor to cardiac disease in this population.

\section{CONCLUSIONS}

This study suggests that subtle cardiac functional abnormalities are present in children and young adults with HIV acquired early in life that are apparent only on newer, loadindependent measures of strain on echocardiography. The years preceding effective ART were characterized by frequent severe cardiac disease. The present study provides preliminary data for subclinical myocardial dysfunction in young individuals with HIV infection. Further studies will be required to confirm this finding and determine its clinical relevance. The present study indicates that, in the era of effective ART and long-term survival with chronic HIV, asymptomatic subclinical cardiac dysfunction may be common.

\section{Abbreviations}

$\begin{array}{ll}\text { ART } & \text { Antiretroviral therapy } \\ \text { HIV } & \text { Human immunodeficiency virus } \\ \text { LV } & \text { Left ventricular } \\ \text { LVMI } & \text { Left ventricular mass index } \\ \text { NIH } & \text { National Institutes of Health }\end{array}$




\section{REFERENCES}

1. Lipshultz SE, Easley KA, Orav EJ, Kaplan S, Starc TJ, Bricker JT, et al. Pediatric Pulmonary and Cardiac Complications of Vertically Transmitted HIV Infection (P2C2 HIV) Study Group. Cardiac dysfunction and mortality in HIV-infected children: the Prospective P2C2 HIV Multicenter Study. Circulation. 2000; 102:1542-1548. [PubMed: 11182983]

2. Luginbuhl LM, Orav EJ, McIntosh K, Lipshultz SE. Cardiac morbidity and related mortality in children with HIV infection. JAMA. 1993; 269:2869-2875. [PubMed: 8388521]

3. Shah I, Prabhu SS, Sumitra V, Shashikiran HS. Cardiac dysfunction in HIV infected children: a pilot study. Indian Pediatr. 2005; 42:146-149. [PubMed: 15767710]

4. Cunha M, Sigueira F, Santos FR, Abreu TF, Oliviera RH, Baptista DM, et al. AIDS in childhood: cardiac involvement with and without triple combination antiretroviral therapy. Arq Bras Cardiol. 2008; 90:11-17. [PubMed: 18317635]

5. Hsue PY, Hunt PW, Ho JE, Farah HH, Schnell A, Hoh R, et al. Impact of HIV infection on diastolic function and left ventricular mass. Circ Heart Fail. 2009; 3:132-139. [PubMed: 19933410]

6. Mondy KE, Gottdiener J, Overton ET, Henry K, Bush T, Conley L, et al. High prevalence of echocardiographic abnormalities among HIV-infected persons in the era of highly active antiretroviral therapy. Clin Infect Dis. 2011; 52:378-386. [PubMed: 21217185]

7. Miller TL, Grant YT, Almeida DN, Sharma T, Lipshultz SE. Cardiometabolic disease in human immunodeficiency virus-infected children. J Cardiometab Syndr. 2008; 3:98-105. [PubMed: 18453810]

8. Lai, W.; Mertens, L.; Ml Cohen; Geva, T. Echocardiography in pediatric and congenital heart disease. London: Wiley-Blackwell; 2009.

9. Marwick TH, Leano RL, Brown J, Sun JP, Hoffmann R, Lysyansky P, et al. Myocardial strain measurement with 2-dimensional speckle-tracking echocardiography: definition of normal range. JACC Cardiovasc Imaging. 2009; 2:80-84. [PubMed: 19356538]

10. Marwick TH. Measurement of strain and strain rate by echocardiography: ready for prime time? J Am Coll Cardiol. 2006; 47:1313-1327. [PubMed: 16580516]

11. D’Hooge J, Heimdal A, Jamal F, Kukulski T, Bijnens B, Rademakers F, et al. Regional strain and strain rate measurements by cardiac ultrasound: principles, implementation and limitations. Eur J Echocardiogr. 2000; 1:154-170. [PubMed: 11916589]

12. Bansal M, Cho GY, Chan J, Leano R, Haluska BA, Marwick TH. Feasibility and accuracy of different techniques of two-dimensional speckle based strain and validation with harmonic phase magnetic resonance imaging. J Am Soc Echocardiogr. 2008; 21:1318-1325. [PubMed: 19041575]

13. Marcus KA, Mavinkurve-Groothuis AM, Barends M, van Dijk A, Feuth T, de Korte C, et al. Reference values for myocardial two-dimensional strain echocardiography in a healthy pediatric and young adult cohort. J Am Soc Echocardiogr. 2011; 24:625-636. [PubMed: 21392941]

14. Amundsen BH, Helle-Valle T, Edvardsen T, Torp H, Crosby J, Lyseggen E, et al. Noninvasive myocardial strain measurement by speckle tracking echocardiography: validation against sonomicrometry and tagged magnetic resonance imaging. J Am Coll Cardiol. 2006; 47:789-793. [PubMed: 16487846]

15. Reant P, Labrousse L, Lafitte S, Bordachar P, Pillois X, Tariosse L, et al. Experimental validation of circumferential, longitudinal, and radial 2-dimensional strain during dobutamine stress echocardiography in ischemic conditions. J Am Coll Cardiol. 2008; 51:149-157. [PubMed: 18191740]

16. Korinek J, Wang J, Sengupta PP, Miyazaki C, Kjaergaard J, McMahon E, et al. Two-dimensional strain - a Doppler-independent ultrasound method for quantitation of regional deformation: validation in vitro and in vivo. J Am Soc Echocardiogr. 2005; 18:1247-1253. [PubMed: 16376750]

17. Brown J, Jenkins C, Marwick TH. Use of myocardial strain to assess global left ventricular function: a comparison with cardiac magnetic resonance and 3-dimensional echocardiography. Am Heart J. 2009; 157(1):102.e1-102.e5. [PubMed: 19081404] 
18. Cho GY, Chan J, Leano R, Strudwick M, Marwick TH. Comparison of two-dimensional speckle and tissue velocity based strain and validation with harmonic phase magnetic resonance imaging. Am J Cardiol. 2006; 97:1661-1666. [PubMed: 16728234]

19. Singh GK, Cupps B, Pasque M, Woodard PK, Holland MR, Ludomirsky A. Accuracy and reproducibility of strain by speckle tracking in pediatric subjects with normal heart and single ventricular physiology: a two-dimensional speckle-tracking echocardiography and magnetic resonance imaging correlative study. J Am Soc Echocardiogr. 2010; 23:1143-1152. [PubMed: 20850945]

20. El-Menyar AA, Galzerano D, Asaad N, Al-Mulla A, Arafa SE, Al Suwaidi J. Detection of myocardial dysfunction in the presence of normal ejection fraction. J Cardiovasc Med (Hagerstown). 2007; 8:923-933. [PubMed: 17906478]

21. Bussadori C, Moreo A, Di Donato M, De Chiara B, Negura D, Dall'Aglio E, et al. A new 2Dbased method for myocardial velocity strain and strain rate quantification in a normal adult and paediatric population: assessment of reference values. Cardiovasc Ultrasound. 2009; 7:8. [PubMed: 19216782]

22. Lopez L, Colan SD, Frommelt PC, Ensing GJ, Kendall K, Younoszai AK, et al. Recommendations for quantification methods during the performance of a pediatric echocardiogram: a report from the Pediatric Measurements Writing Group of the American Society of Echocardiography Pediatric and Congenital Heart Disease Council. J Am Soc Echocardiogr. 2010; 23:465-495. [PubMed: 20451803]

23. Koopman LP, Slorach C, Manlhiot C, McCrindle BW, Jaeggi ET, Mertens L, et al. Assessment of myocardial deformation in children using Digital Imaging and Communications in Medicine (DICOM) data and vendor independent speckle tracking software. J Am Soc Echocardiogr. 2011; 24:37-44. [PubMed: 21095099]

24. Blessberger H, Binder T. Two dimensional speckle tracking echocardiography: basic principles. Heart. 2010; 96:716-722. [PubMed: 20424157]

25. Cho GY, Marwick TH, Kim HS, Kim MK, Hong KS, Oh DJ. Global 2-dimensional strain as a new prognosticator in patients with heart failure. J Am Coll Cardiol. 2009; 54:618-624. [PubMed: 19660692]

26. Lipshultz SE, Shearer WT, Thompson B, Rich KC, Cheng I, Orav EJ, et al. Cardiac effects of antiretroviral therapy in HIV-negative infants born to HIV-positive mothers: NHLBI CHAART-1 (National Heart, Lung, and Blood Institute Cardiovascular Status of HAART Therapy in HIVExposed Infants and Children cohort study). J Am Coll Cardiol. 2010; 57:76-85. [PubMed: 21185505]

27. Ho JE, Hsue PY. Cardiovascular manifestations of HIV infection. Heart. 2009; 95:1193-1202. [PubMed: 19564432]

28. Fisher SD, Easley KA, Orav EJ, Colan SD, Kaplan S, Starc TJ, et al. Mild dilated cardiomyopathy and increased left ventricular mass predict mortality: the prospective P2C2 HIV Multicenter Study. Am Heart J. 2005; 150:439-447. [PubMed: 16169321]

29. Wang X, Chai H, Lin PH, Yao Q, Chen C. Roles and mechanisms of human immunodeficiency virus protease inhibitor ritonavir and other anti-human immunodeficiency virus drugs in endothelial dysfunction of porcine pulmonary arteries and human pulmonary artery endothelial cells. Am J Pathol. 2009; 174:771-781. [PubMed: 19218343]

30. Choi AI, Vittinghoff E, Deeks SG, Weekley CC, Li Y, Shlipak MG. Cardiovascular risks associated with abacavir and tenofovir exposure in HIV-infected persons. AIDS. 2011; 25:12891298. [PubMed: 21516027]

31. Helle-Valle T, Crosby J, Edvardsen T, Lyseggen E, Amundsen BH, Smith HJ, et al. New noninvasive method for assessment of left ventricular rotation: speckle tracking echocardiography. Circulation. 2005; 112:3149-3156. [PubMed: 16286606] 
(A)

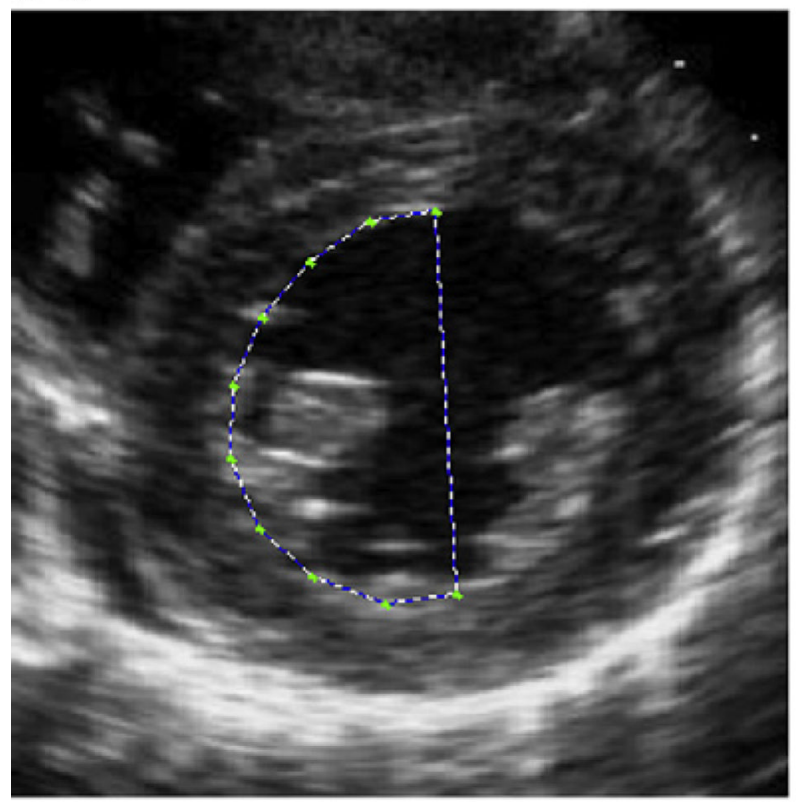

(B)

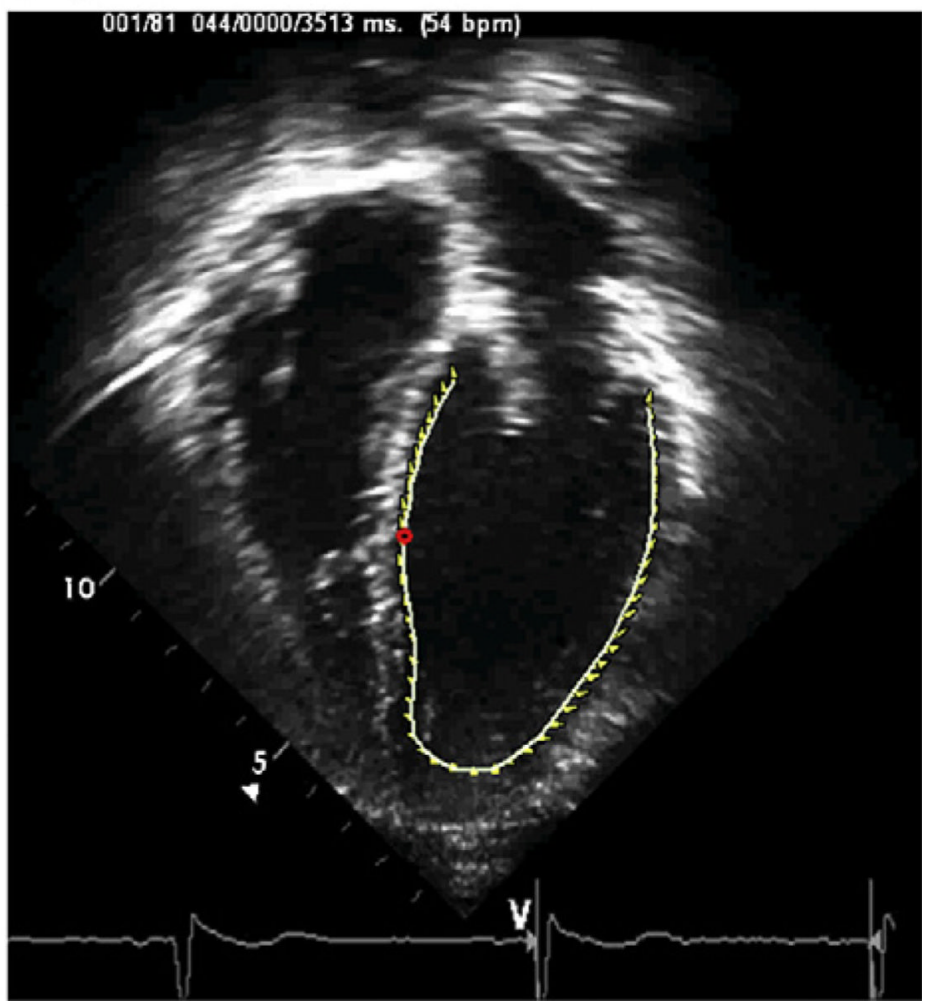

Figure 1.

(A) Short-axis speckle-tracking imaging (circumferential and radial strain). (B) Apical fourchamber speckle-tracking imaging (longitudinal strain). 


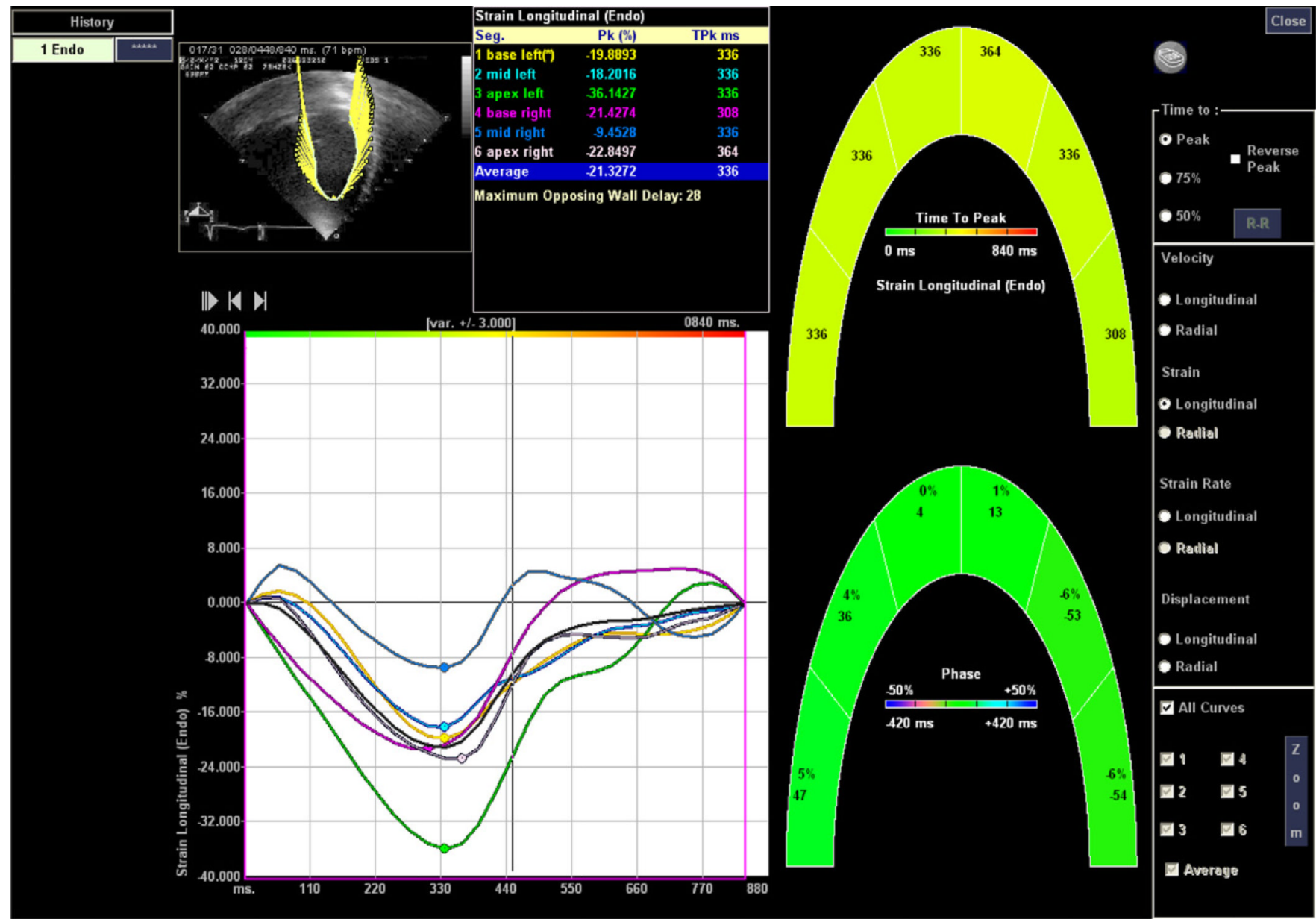

Figure 2.

Longitudinal speckle-tracking strain imaging. 
A

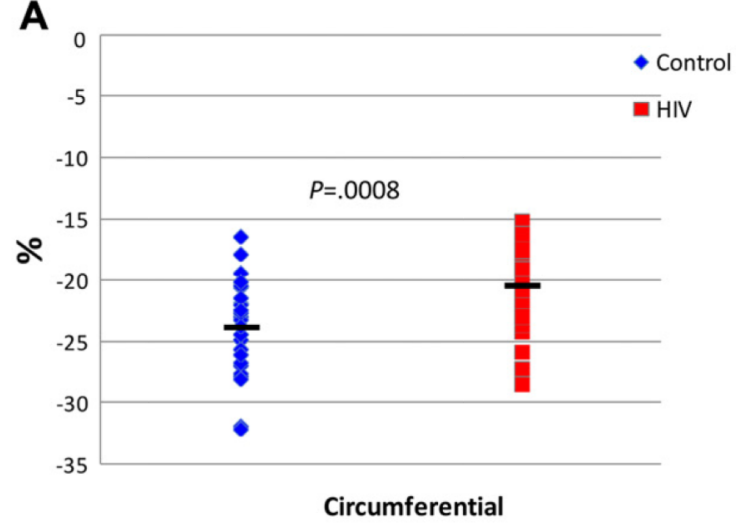

C
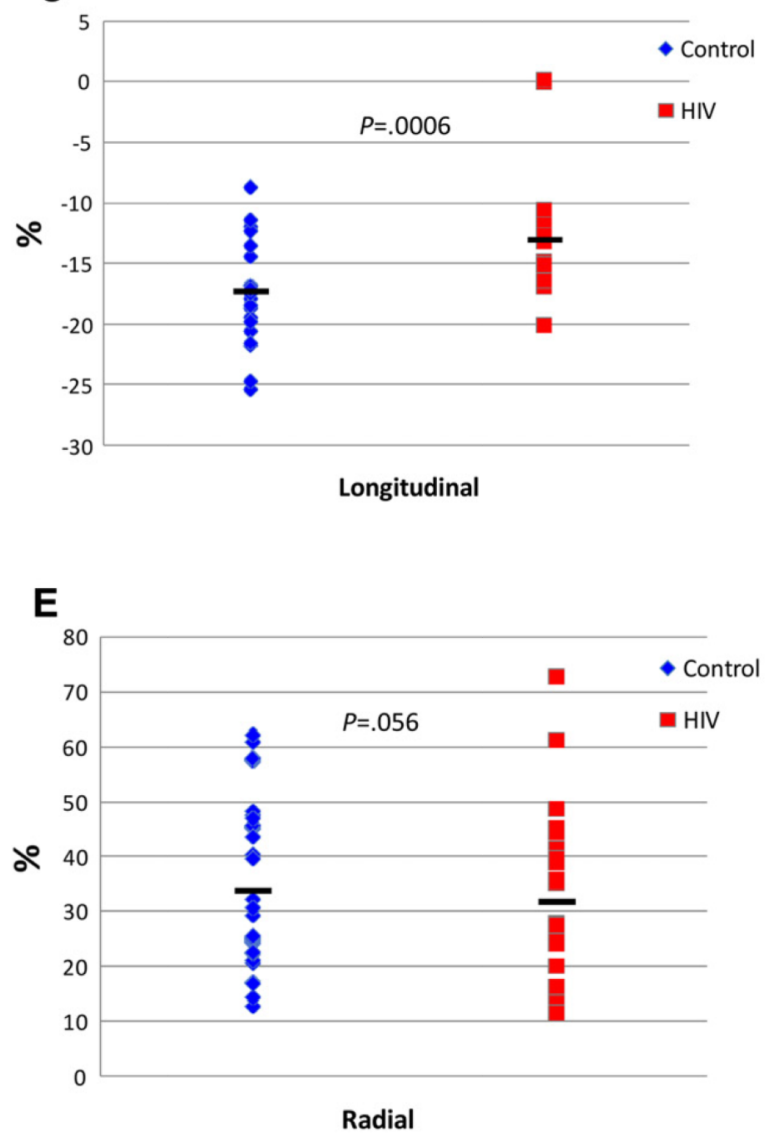

B

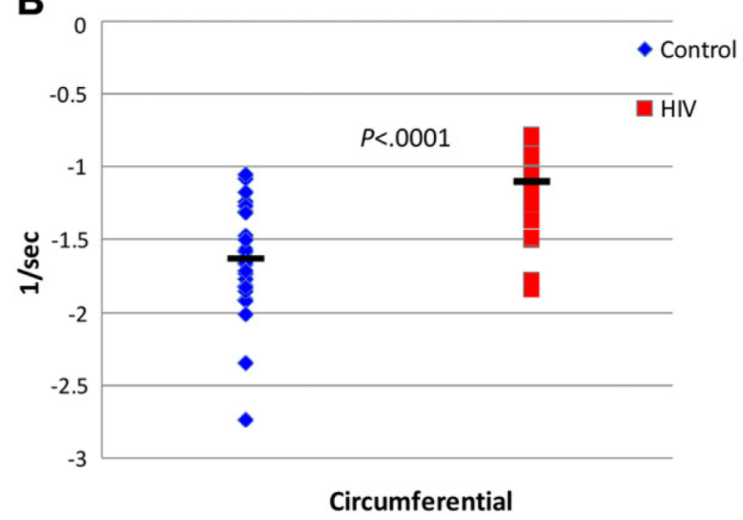

D

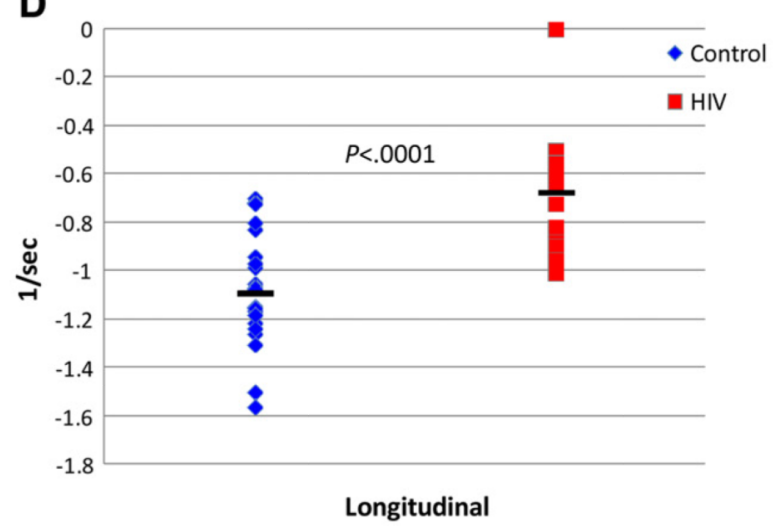

$\mathbf{F}$

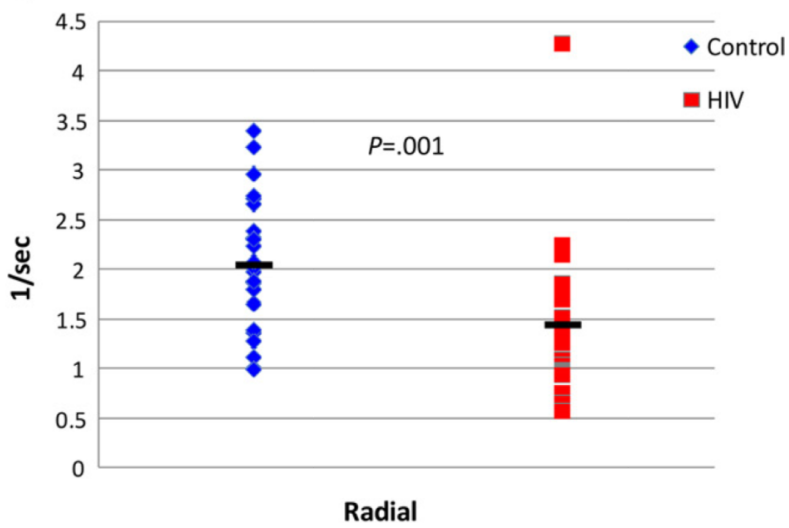

Figure 3.

Longitudinal, circumferential, and short-axis radial percentage cardiac strain between HIVinfected subjects (red squares) and matched controls (blue diamonds) $(\mathbf{A}, \mathbf{C}$, and $\mathbf{E}$, respectively). Longitudinal, circumferential, and short-axis radial cardiac strain rate between HIV-infected subjects and matched controls $(\mathbf{B}, \mathbf{D}$, and $\mathbf{F}$, respectively). Black bars indicate mean values, and $P$ value represent between-group differences by Wilcoxon's rank-sum test. 


\section{Table 1}

Demographics, traditional echocardiographic measurements, and HIV characteristics

\begin{tabular}{|c|c|c|c|}
\hline Variable & $\begin{array}{l}\text { HIV positive } \\
\quad(n=28)\end{array}$ & $\begin{array}{l}\text { Control } \\
(n=28)\end{array}$ & $P$ \\
\hline Age (y) & $18 \pm 4$ & $19 \pm 5$ & .80 \\
\hline Male/female & $12 / 16$ & $12 / 16$ & 1.00 \\
\hline African American & $46 \%$ & $39 \%$ & .60 \\
\hline BMI $\left(\mathrm{kg} / \mathrm{m}^{2}\right)$ & $23 \pm 4$ & $23 \pm 4$ & .30 \\
\hline $\operatorname{BSA}\left(m^{2}\right)$ & $1.6 \pm 0.2$ & $1.7 \pm 0.2$ & .40 \\
\hline Systolic BP (mm Hg) & $121 \pm 12$ & $116 \pm 11$ & .07 \\
\hline Diastolic BP (mm Hg) & $72 \pm 8$ & $67 \pm 2$ & .06 \\
\hline \multicolumn{4}{|l|}{ Echocardiography } \\
\hline Ejection fraction $(\%)$ & $61 \pm 4$ & $62 \pm 4$ & .30 \\
\hline Shortening fraction $(\%) *$ & $36.7 \pm 4.1$ & $34 \pm 3.8$ & .06 \\
\hline $\operatorname{LVMI}\left(\mathrm{g} / \mathrm{m}^{2}\right)^{*}$ & $81.6 \pm 17.1$ & $73.3 \pm 12.3$ & .06 \\
\hline LV mass $Z$ score ${ }^{*}$ & $-0.51 \pm 1.11$ & $-1.04 \pm 0.61$ & .046 \\
\hline E/A ratio & $2 \pm 0.5$ & $1.8 \pm 0.5$ & .20 \\
\hline $\mathrm{E} / \mathrm{E}^{\prime}$ ratio (lateral) & $5 \pm 1.2$ & $5.2 \pm 1.8$ & .80 \\
\hline $\mathrm{E} / \mathrm{E}^{\prime}$ ratio (septal) & $6.5 \pm 2.1$ & $7.2 \pm 1.9$ & .30 \\
\hline Tricuspid regurgitation velocity $(\mathrm{m} / \mathrm{sec})$ & $2.1 \pm 0.3$ & $2.1 \pm 0.2$ & .80 \\
\hline LV dimension $(\mathrm{mm})$ & $4.6 \pm 0.5$ & $4.7 \pm 0.5$ & .50 \\
\hline \multicolumn{4}{|l|}{ HIV characteristics and lipids } \\
\hline Cumulative ARV exposure (y) & $14.6 \pm 3.1$ & - & - \\
\hline Cumulative PI exposure (y) & $9.9 \pm 2.8$ & - & - \\
\hline Cumulative NRTI exposure (y) & $3.7 \pm 3.7$ & - & - \\
\hline Median CD4 count (cells/mm $\left./ \mathrm{mm}^{3}\right)$ & 667 & - & - \\
\hline Viral load < 50 copies $/ \mathrm{mL}$ & $71 \%$ & - & - \\
\hline HDL cholesterol (mg/dL) & $45 \pm 13$ & - & - \\
\hline Triglycerides (mg/dL) & $150 \pm 170$ & - & - \\
\hline Total cholesterol (mg/dL) & $156 \pm 36$ & - & - \\
\hline
\end{tabular}

$B M I$, Body mass index; $B P$, blood pressure; $B S A$, body surface area; $H D L$, high-density lipoprotein; $N R T I$, nucleoside reverse transcriptase inhibitor; PI, protease inhibitor.

Data are expressed as mean $\pm \mathrm{SD}$ or as numbers or percentages.

For the HIV-positive and control groups, $n=15$ and $n=25$, respectively. 
Table 2

Assessment of intraobserver and interobserver variability with intraclass correlation coefficients

\begin{tabular}{lcc}
\hline Variable & $\begin{array}{c}\text { Intraobserver } \\
\text { variability }(\boldsymbol{n}=\mathbf{5 3})\end{array}$ & $\begin{array}{c}\text { Interobserver } \\
\text { variability }(\boldsymbol{n}=\mathbf{1 0})\end{array}$ \\
\hline Circumferential strain & $0.833(0.73-0.90)$ & $0.836(0.49-0.96)$ \\
\hline Circumferential strain rate & $0.935(0.89-0.96)$ & $0.855(0.54-0.96)$ \\
\hline Radial strain & $0.534(0.30-0.71)$ & $0.815(0.45-0.95)$ \\
\hline Radial strain rate & $0.488(0.25-0.67)$ & $0.859(0.56-0.96)$ \\
\hline Longitudinal strain & $0.859(0.76-0.92)$ & $0.857(0.52-0.97)$ \\
\hline Longitudinal strain rate & $0.921(0.86-0.96)$ & $0.860(0.53-0.96)$ \\
\hline
\end{tabular}

Data are expressed as intraclass correlation coefficient (95\% confidence interval). 